\title{
Compactness in probabilistic Hilbert space
}

Waffa faeik keidan

\section{Compactness in probabilistic Hilbert space}

\author{
Waffa faeik keidan \\ Diyala University - Mathematic department \\ Received 16 June 2016 ; Accepted 4 September 2016
}

\begin{abstract}
$\underline{\text { Abstract }}$
In this paper we focus our study on weakly, strongly and uniformly convergence of operators in probabilistic Hilbert space (PH- space) and the relations between these convergence of operators. Also, we introduce the definition of operator in ( $\mathrm{PH}-$ space).
\end{abstract}

Keyword: compact operator, PH- space, weakly convergence, strongly, convergence, bounded operator.

$$
\begin{aligned}
& \text { التر اص في فضاء هلبرت الاحتمالي } \\
& \text { وفاء فايق غيدان } \\
& \text { جامعة ديالى - كلية العلوم }
\end{aligned}
$$

$$
\begin{aligned}
& \text { الخلاصة } \\
& \text { في هذا البحث تركزت در استنا حول تقارب المؤثرات في فضاء هلبرت الاحتمالي ( التقارب الضعيف، القوي، المنتظم) } \\
& \text { و العلاقة بين المؤثرات المتقاربة. كذللك قدمنا المؤثر المتراص في فضناء هلبرت الاحتمالي. } \\
& \text { الكلمات المفتاحية: المؤثر المتر اص، فضاء هلبرت الاحتمالي، التقارب الضعيف، التقارب القوي، المؤثر المحدود }
\end{aligned}
$$




\section{Compactness in probabilistic Hilbert space}

\section{Waffa faeik keidan}

\section{$\underline{\text { Introduction }}$}

The concept of a probabilistic inner product space ( PIP-space) which is based on the modern concept of a PH- space that was newly introduced in [01,02,03]. The concept of PH- space was also introduced and studied in 2007 by S.Y.; wany, $x$, : and Ga, J. [04] . Before we proceed we must state some definitions. Known details and results to be used in the complement the concepts used are those of [01,05]

\section{Definition 1 [01]}

In probability theory, a distribution function (D.F) is a mapping $G: R \rightarrow[0,1]$ check the following:

1 - $\mathrm{G}$ is non-decreasing.

2- $\mathrm{G}$ is left-continuous.

3- $\inf _{x \in R} G(X)=0$.

4- $\sup _{x \in R} G(x)=1$

\section{Note:}

1- D: The set of all D.F's .

2- $H(x)=\left\{\begin{array}{lll}0 & \text { if } & x \leq 0 \\ 1 & \text { if } & x>0\end{array}\right.$

3- $H \in D$ (special element)

\section{Definition 2 [04]}

A PIP-space is a triple $(E, G, *)$ whese: $E$ is a real linear space, And $\mathrm{G}$ is a mapping of $E \times$ $E \rightarrow D\left(G_{u, v}\right.$ will denote the D.F), where: $G_{u, v}(x)$ will represent the value $G_{u, v}$ at $x \in$ $R$ satisfies the following conditions:

$$
\begin{aligned}
& \text { 1- } G_{u, u}(0)=0, \quad \forall u \in E . \\
& \text { 2- } G_{u, v}(x)=G_{v, u}(x), \quad \forall u, v \in E .
\end{aligned}
$$




\section{Compactness in probabilistic Hilbert space}

\section{Waffa faeik keidan}

3- $G_{u, v}(x)=H(x)$ if and only if $u=0$

4- For $u, v \in E$ and $\gamma \in R$

$$
G_{\gamma u, v}(x)= \begin{cases}G_{u, v}\left(\frac{x}{\gamma}\right) & \gamma>0 \\ H(x) & \gamma=0 \\ 1-G_{u, v}\left(\frac{x}{\gamma}+\right) & \gamma<0\end{cases}
$$

Where $G_{u, v}(x+)=\lim _{x_{1} \rightarrow x+} G_{u, v}\left(x_{1}\right)$

5- If $u$ and $v$ is linearly independent then

$$
G_{u+v, w}(x)=\left(G_{u, w} * G_{w, v}\right)(x)
$$

Where $\left(G_{u, v} * G_{W, v}\right)=\int_{-\infty}^{+\infty} G_{u, W}(x-t) d G_{w, v}(t)$

\section{Definition 3 [04]}

A sequence $u_{n}$ in $E$ is said be $\tau-$ converges to $u \in E$ if $\forall \epsilon>0$ and $\propto>0$ there must exists $N(\epsilon, \propto)$ appositive integer s.t

$$
G_{u_{n}-u, u_{n}-u}(\epsilon)>1-\propto \text { whenever } n>N
$$

\section{Definition 4 [04]}

A sequence $u_{n}$ in $E$ is said be $\tau-$ cauchy sequence if $\forall \epsilon>0$ and $\propto>0$ there must exists $N(\epsilon, \propto)$ s.t

$$
G_{u_{n}-u_{m}, u_{n}-u_{m}}(\epsilon)>1-\propto \text { whenever } n, m>N
$$

\section{Lemma $1[06]$}

Let $G(x)$ be $\mathrm{d}$ f, Assume $g(x)$ is non decreasing bounded function then

$$
\int_{-\infty}^{+\infty} g(x) d G(x)=\int_{-\infty}^{+\infty} g(x) d G(x+)
$$




\section{Compactness in probabilistic Hilbert space}

\section{Waffa faeik keidan}

\section{Definition 5 [06]}

A PIP- space $(E, G, *)$ is called with mathematical exaction if

$\int_{-\infty}^{+\infty} x d G_{u, v}(x)$

Is converges for $u, v \in E$.

\section{$\underline{\text { Remake }}$}

$(E, G, *)$ is said to be $(\tau-$ cauch sequence in $E) \Rightarrow(\tau-$ converges to some point in $E)$

\section{Theorem 1 [06]}

Let $(E, G, *)$ be PIP- space with (ME) letting

$$
<u, v>=\int_{-\infty}^{+\infty} x d G_{u, v}(x)
$$

$\forall x, y \in E$, Then $\left(E,<_{.}, .>\right)$is IP-space, so that $(E,\|\|$.$) is a nor med space, where \|u\|=$ $\sqrt{<u, u>}$ for all $u \in E$

\section{Weak convergence}

\section{Definition 1}

Let $G_{n}$ and $G$ are dF's $G_{n} \stackrel{\text { weakly }}{\longrightarrow} G$ if $G_{n}(x) \longrightarrow G(x)$ for every point at which the timid df $G$ is continuous if

$$
C(G)=\{x \in R: G(x)=G(x+0)\}
$$

$\forall x \in C(G)$

$$
\lim _{n \rightarrow \infty} G_{n}(x)=G(x)
$$




\section{Compactness in probabilistic Hilbert space}

\section{Waffa faeik keidan}

\section{Definition 2}

Let $(E, G, *)$ be PH- space, and $u_{n}, u \in E$ :

(1) $u_{n} \stackrel{\text { weakly }}{\longrightarrow} u$ if $v \in E$, we have $\lim _{n \rightarrow \infty}\left\langle u_{n}, v\right\rangle=\langle u, v\rangle$

(2) $u_{n} \stackrel{N}{\longrightarrow} u$ (N-convergent) if $\lim _{n \rightarrow \infty} G_{u_{n}-u, u_{n}-u}(x)=1$

\section{Remark 1}

(1) If $u_{n} \stackrel{w}{\longrightarrow} u$ then $u_{n} \stackrel{m}{\longrightarrow} u$ (m-convergent)

(2) If $u_{n} \stackrel{w}{\longrightarrow} u$ then $u_{n} \stackrel{\tau}{\longrightarrow} u$ ( $\tau$-convergent $)$

\section{Lemma 1}

Let $(E, G, *)$ PH- space, and $u_{n} \in E$. If $\left\|u_{n}-u\right\| \rightarrow 0$ then $u_{n} \stackrel{w}{\longrightarrow} u$

\section{$\underline{\text { Proof }}$}

Since $\left\|u_{n}-u\right\| \rightarrow 0 \Rightarrow\left\|u_{n}-u\right\|^{2} \rightarrow 0$

$$
\begin{aligned}
& \lim _{n \rightarrow \infty} \int_{0}^{\infty} x d G_{u_{n}-u, u_{n}-u}(x)=0 \\
& \lim _{n \rightarrow \infty} G_{u_{n}-u, u_{n}-u}(x)=0 \\
& \lim _{n \rightarrow \infty}\left(u_{n}-u\right)=0 \\
& \lim _{n \rightarrow \infty} u_{n}=u
\end{aligned}
$$

\section{Lemma 2}

Let $u_{n} \stackrel{w}{\longrightarrow} u$ then $u_{n}$ is bounded. 


\section{Compactness in probabilistic Hilbert space}

\section{Waffa faeik keidan}

\section{Proof}

For any $v \in E$, we have

$\lim _{n \rightarrow \infty}\left\langle u_{n}, v>=\langle u, v>, u \in E\right.$

$\lim _{n \rightarrow \infty} \int_{0}^{\infty} x d G_{u_{n}, v}(x)=\int_{0}^{\infty} x d G_{u, v}(x)$

Since ever $G_{u_{n, v}}$ is bounded $0 \leq G_{u_{n}, v}(x) \leq 1 \quad \forall x \in \bar{R}$

Therefore the sequence $\left\{\left\langle u_{n}, v\right\rangle\right\}$ is bounded. Now the lemma follows for the principle of uniform boundedness.

\section{Lemma 3}

Let $(E, G, *)$ be PH- space, and $u_{n}, v_{n} \in E$ such that:

$u_{n} \stackrel{w}{\longrightarrow} u$ and

$\lim _{n \rightarrow \infty} u_{n}=v, v \in E$ then

$\left.\lim _{n \rightarrow \infty}<u_{n}, v_{n}\right\rangle=\langle u, v\rangle$

Or $<u_{n}, v_{n}>\stackrel{w}{\longrightarrow}<u, v>$

\section{Proof}

Since any convergent sequence is bounded, the in equality

$$
\begin{gathered}
\left|<u_{n}, v_{n}>-<u, v>\right|=\left|<u_{n}-u, v_{n}>+<u, v_{n}-v>\right| \\
\leq\left\|u_{n}-u\right\|\left\|v_{n}\right\|+\|u\|\left\|v_{n}-v\right\|
\end{gathered}
$$




\section{Compactness in probabilistic Hilbert space}

\section{Waffa faeik keidan}

Implies that

$$
\begin{aligned}
& \lim _{n \rightarrow \infty} \int_{0}^{\infty} x d G_{u_{n}, v_{n}}(x)-\int_{0}^{\infty} x d G_{u, v}(x)=0 \\
& \lim _{n \rightarrow \infty} \int_{0}^{\infty} x d G_{u_{n}, v_{n}}(x)=\int_{0}^{\infty} x d G_{u, v}(x)=0 \\
& \lim _{n \rightarrow \infty}\left\langle u_{n}, v_{n}\right\rangle=\langle u, v\rangle
\end{aligned}
$$

\section{Theorem 1}

Let $(E, G, *)$ be PH- space with ME, left $T: D(T) \rightarrow R(T)$. linear bounded operator, then these exists $\mu>0$ s.t

$$
|<T u, v>| \leq\|T u\|\|v\|
$$

$$
\leq \mu\|u\|\|v\| \quad \forall u, v \in E
$$

\section{Proof}

Fix $v \in E$ and $\varphi_{v}(u)=\left\langle T u, v>, \forall u \in E \varphi_{v}\right.$ is linear functional on $E$, i.e $\varphi_{v}: E \rightarrow R$

For any positive real number $\propto$

1) If $T u-T v$ with $u-v$ is linearly independent, then $T u-\propto I v \neq 0$ then

$$
\begin{aligned}
& <T u-\propto I v, T u-\propto I v>=\int_{-\infty}^{+\infty} x d G_{T u-\propto I v, T u-\propto I v}(x) \geq 0 \\
& =\int_{-\infty}^{+\infty} x d G_{T u, T u}(x)-\int_{-\infty}^{+\infty} x d G_{T u, \propto I v}(x)-\int_{-\infty}^{+\infty} x d G_{\propto I v, T u}(x)+\int_{-\infty}^{+\infty} x d G_{\propto v, \propto v}(x) \geq 0 \\
& =\int_{-\infty}^{+\infty} x d F_{T u, T u}(x)\left|+2 \propto \int_{-\infty}^{+\infty} x d F_{T u, v}(x)\right|+\propto^{2} \int_{-\infty}^{+\infty} x d F_{v, v}(x) \geq 0 \\
& \|T u\|^{2}-2 \propto|<T u, v>|+\propto^{2}\|v\|^{2} \geq 0
\end{aligned}
$$

Let $\propto=\frac{|<T u, v>|}{\|v\|^{2}}$ 


\section{Compactness in probabilistic Hilbert space}

\section{Waffa faeik keidan}

$\|T u\|^{2}-2 \frac{|<T u, v>|^{2}}{\|v\|^{2}}+\frac{|<T u, v>|^{2}}{\|v\|^{2}} \geq 0$

$\|T u\|^{2}-\frac{|<T u, v>|^{2}}{\|v\|^{2}} \geq 0$

$|<T u, v>|^{2} \leq\|T u\|^{2}\|v\|^{2}$

$|<T u, v>| \leq\|T u\|\|v\|$

Since $T$ bounded in norm

$|<T u, v>| \leq \mu\|u\|\|v\|$

2) If $T u-T v$ with $u-v$ is linearly depended and $T u-\propto I v \neq 0$, then prove is same in (1).

3) If $T u-T v$ with $u-v$ is linearly depended and $T u-\propto I v=0$ then

$<T u-\propto I v, T u-\propto I v>=\int_{-\infty}^{+\infty} x d G_{T u-\propto I v, T u-\propto I v}(x)$

$$
=\int_{-\infty}^{+\infty} x d H(x)=0
$$

$|<T u, v>| \leq\|T u\|\|v\|$

$\leq \mu\|x\|\|u\|$

$\forall u, v \in E$ and $\mu>0$

\section{Theorem 2}

If $T$ is bounded operator then $\|T\|=\sup _{\|u\|=1}|<T u, u>|$

\section{Proof}

For any $u \in E$

$<T u, u>=<u, u T>$ is real 


\section{Compactness in probabilistic Hilbert space}

\section{Waffa faeik keidan}

Now let

$M=\sup _{\|u\|=1}|<T u, u>|$

By lemma 1

$|<T u, v>| \leq\|T u\|\|u\|$

$$
\leq\|T\|\|u\|^{2}=\|T\|
$$

For all $u \in E$ such that $\|u\|=1$. Consequently $M \leq\|T\|$

On the other hand, for any $t, s, \in E$ we have

$$
\begin{aligned}
4<T t, s>= & 4 \int_{-\infty}^{+\infty} x d G_{T t, s}(x) \\
& =\int_{-\infty}^{+\infty} x d G_{T(t+s), t+s}(x)-\int_{-\infty}^{+\infty} x d G_{T(t-s), t-s}(x) \\
& \leq M\left(\|t+s\|^{2}+\|t-s\|^{2}\right) \\
& =2 M\left(\|t\|^{2}+\|s\|^{2}\right)
\end{aligned}
$$

Using the parallelogram law. If $T t \neq 0$ let $s=\frac{\|t\|}{\|T t\|} T u$ to obtain, since $\|t\|=\|s\|$ then $\|t\|\|T t\| \leq M\|t\|^{2}$

Consequently $\|T t\| \leq M\|t\| \quad$ (for all $u$, in clouding these with $T t=0$ ) and $\|T\| \leq M$

Therefore $\|T\|=M$.

\section{Definition 3}

Let $T_{n}$ be a sequence of bounded operator in $E$.

i) $\quad T_{n}$ uniformly convergent to $T$ if: 


\section{Compactness in probabilistic Hilbert space}

\section{Waffa faeik keidan}

$$
\lim _{n \rightarrow \infty} \int_{0}^{\infty} x d G_{T_{n}-T, T_{n}-T}(x)=0
$$

ii) $\quad T_{n}$ strongly convergent to $T$ if :

$$
\lim _{n \rightarrow \infty} \int_{0}^{\infty} x d G_{T_{n} u-T u, T_{n} u-T u}(x)=0 \quad, \quad u \in E
$$

iii) $\quad T_{n} \stackrel{w}{\longrightarrow} T$ if:

$$
\lim _{n \rightarrow \infty} \int_{0}^{\infty} x d G_{T_{n} u, v}(x)=\quad \int_{0}^{\infty} x d G_{T_{n} u, v}(x) \quad, \quad \forall u, v \in E
$$

\section{Compact Operators}

A linear operator $T$ is said to be compact if the image of any bounded sequences has a convergent of subsequences.

\section{Theorem 1}

Let $(E, G, *)$ be probabilistic Hilbert space with mathematical expection, let $T: D(T) \rightarrow R(T)$. linear bounded operator in $(E, G, *), T$ is compact operator.

\section{$\underline{\text { Proof }}$}

For every sequence $u_{n} \in E$ such that $\left\|u_{n}\right\|=1$ then by theorem (2-2)

$\|T\|=\sup _{\|u\|=1}\left|<T u_{n}, u_{n}>\right|$

Then

$\lim _{n \rightarrow \infty}\left|<T u_{n}, u_{n}>\right|=\|T\|$

Let $v_{n}=T u_{n}$ has a convergent subsequences related this subsequences as $u_{n}$ and let $v=\lim _{n \rightarrow \infty} T u_{n}$ 


\section{Compactness in probabilistic Hilbert space}

\section{Waffa faeik keidan}

$$
\begin{aligned}
& \lim _{n \rightarrow \infty}<T u_{n}, u_{n}>=\mp\|T\|=\lambda \\
& \begin{aligned}
\left\|T u_{n}-\lambda u_{n}\right\|^{2} & =\int_{-\infty}^{+\infty} x d G_{T_{n} u-\lambda u_{n}, T_{n} u-\lambda u_{n}}(x) \\
& =\int_{-\infty}^{+\infty} x d G_{T_{n} u, T_{n} u}(x)-2 \lambda \int_{-\infty}^{+\infty} x d G_{T_{n} u, \lambda u_{n}}(x)+\lambda^{2} \int_{-\infty}^{+\infty} x d G_{u_{n}, u_{n}}(x)
\end{aligned}
\end{aligned}
$$

Since $\int_{-\infty}^{+\infty} x d G_{u_{n}, u_{n}}(x)=\left\|u_{n}\right\|^{2}=1$

$$
\begin{aligned}
\left\|T u_{n}-\lambda u_{n}\right\|^{2} & =\left\|T u_{n}\right\|^{2}-2 \lambda<T u_{n}, u>+\lambda^{2} \\
& \leq 2 \lambda^{2}-2 \lambda<T u_{n}, u_{n}>
\end{aligned}
$$

Since $<T u_{n}, u_{n}>\rightarrow \lambda$

$\left\|T u_{n}-\lambda u_{n}\right\|^{2} \rightarrow 0$

Then

$T u_{n}-\lambda u_{n} \rightarrow 0$

On the other hand $T u_{n} \rightarrow v$ and consequently $u_{n}$ also converges :

$u_{n} \rightarrow u=\lambda^{-1} v \quad u_{n}$ convergent to $u$.

$\mathrm{T}$ is compact operator. 


\section{Compactness in probabilistic Hilbert space \\ Waffa faeik keidan}

\section{$\underline{\text { References }}$}

1. C.ALSINA-B SCHWEIZER-A. SKLAR: On the definition of a probabilistic normed space. A equations math, 48(1993), 91-98.

2. C.ALSINA-B SCHWEIZER-A. SKLAR: continuity properties of probabilistic norms J.math. Anal. Appl. 208(1997) on. 2, 446-452.

3. B.LAFUERZA GUILLEN- J.A. RODRIGUEZ LALLENA-C. SEMPI: Some classes of probabilistic normed spaces, (to appear).

4. SU, Y. ; Wany ,X.; and Gao, J.(2007). Riesz Theorem in probabilistic lnner product spaces, International mathematical form, pp: 3073-3078.

5. B. Schweizer and A. Sklor, probabilistic metric spaces, North-Holland, New York, 1983.

6. Y.F.SU, X.Z. waug, and J.Y. Gao :Approximation of fixed points for non linear operators in probabilistic inner produced spaces, Int. J. of Appl. Math. And mech. 4(4):1-9,2008. 